\title{
VIBRATION CONTROL USING PIEZOELECTRIC CONNECTED WITH SHUNTS CIRCUITS
}

\author{
BRAION B. MOURA ${ }^{1}$ AND MARCELA R. MACHADO ${ }^{2}$ \\ ${ }^{1}$ University of Brasília, Department of Mechanical Engineering \\ Brasília-DF, Brazil, 70910-900 \\ braionmoura@gmail.com,marcelam@unb.br \\ ${ }^{2}$ University of Brasília, Department of Mechanical Engineering \\ Brasília-DF, Brazil, 70910-900 \\ marcelam@unb.br
}

Key words: Smart Material, Piezoelectric, Shunt, Spectral Element Method

\begin{abstract}
The study analyzes the dynamic behaviour and vibration attenuation of an Euler Bernoulli beam coupled with a piezoelectric layer. The vibration attenuation is given by the shunt circuit connected to the piezoelectric. This paper shows different shunt circuit impedances and how they affect the system's frequency response (FRF) functions. The spectral element method is used to model the intelligent material under analysis and has its function based on the exact solution of the wave equation, therefore, a single element is necessary to model the structure. The estimated responses for each shunt configuration express a relationship with the passive components used in the respective circuits. In each case, the circuit has a particular effect on FRFs.
\end{abstract}

\section{INTRODUCTION}

Smart materials are increasingly present in vibrational control studies and application. Those kinds of materials use piezoelectrics (PZT) to stand out for the feasibility of adjusting vibration attenuation and control. This potentiality from PZTs can be obtained through passive, active and hybrid control [25]. The control of unwanted vibrations and noise can be performed with passive control PZT shunt circuit [26]. This control deals with a direct piezoelectric effect that modifies the structure's rigidity and damping properties with the addition of circuits with different combinations of resistive, inductive, capacitive elements, and switches [11]. The piezoelectric materials allow the emergence of said intelligent materials, because when changes occur in the state variables in a mechanical, physical domain [20]. They cause a difference in the state variables in a physical, electrical domain and vice versa [4]. The use of smart material gained renewed interest in applications involving vibration-based, energy harvesting, nonlinearities, and in the framework of metamaterials $[12,14]$. Smart metamaterials can result in adaptive and multifunctional metamaterials.

Passive control uses external shunts circuits coupled to PZTs, which can perform vibrational without the external power supply. Forward [10] was the pioneers in associate some piezoelectric shunts circuits with the systems to induce vibrational control. Despite the wide variety of circuit topographies and their respective effects, a contribution to resistive and inductive shunts was discussed by Hagood and Flotow 
[13]. The purely resistive shunts were related to structural damping bands, and resistive inductive circuits were related to controlling modes of vibration, similar to a dynamic absorber. Shunt circuit configurations that involve capacitive elements denote changes in the natural frequencies of the coupled system [5]. Periodicity relations that involve techniques such as multi-mode [1], negative capacitance [17, 24] and variations in the RLC circuit [16], are known to induce bandgap effects on vibrating structures. Another variation for these capacitive, resistive and inductive shunt configurations is in the switching element, controlling the piezoelectric's current and voltage flow.

PZT shunts to promote vibrational control can be simulated via the finite element method (FEM), which requires a large number of elements in the mesh $[3,11]$. An alternative to FEM in dynamic simulation and analysis is the spectral element method (SEM). This method is formulated from the wave equation's analytical solution, which reduces the number of elements in the simulation significantly $[9,18]$. Therefore, a single element is sufficient to model a smart material with a uniform section along its length. A few spectral elements have been developed, like as rod, beam, plates, cables, and ongoing researches are proposing new and improved elements $[6,7,8,9,18]$. Lee and Kim [19, ?] developed a spectral element coupling the Euler-Bernoulli beam with a piezoelectric layer, and active piezoelectric. The smart beam spectral element with passive control was proposed and demonstrated in $[2,21,22,23]$. In this work, the objective is to use SEM to model a smart material consisting of an aluminium beam coupled to a PZT layer connected to the shunt circuit in the configuration of resistive, inductive, capacitive, and variations. The shunts circuits can affect the dynamic response of a beam. The results showed the circuits impedance curve and the influence over the FRFs.

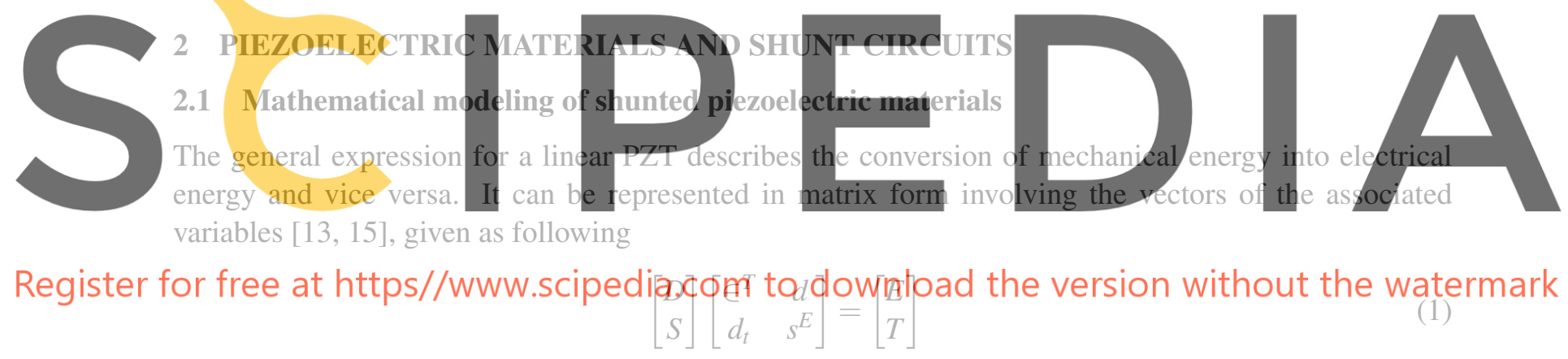

where $D$ is the vector of electrical displacement $\left(C / \mathrm{m}^{2}\right), T$ is the vector of material stresses $\left(\mathrm{N} / \mathrm{m}^{2}\right), E$ is the vector of electrical field $(V / m), S$ is the vector of material strains, $\in^{T}$ is the matrix with dielectric constants, $s^{E}$ is the the compliance matrix of the piezoceramic, and $d$ is the matrix of piezoelectric constants. Applying the Laplace transform in Eq. 1 and using current $I$ and voltage $V$ relationships to eliminate terms $E$ and $D$, the following general equation for a PZT is obtained

$$
\left[\begin{array}{l}
I \\
S
\end{array}\right]\left[\begin{array}{cc}
Y^{E L} & s A d \\
d_{t} L^{-} 1 & s^{E}
\end{array}\right]=\left[\begin{array}{l}
V \\
T
\end{array}\right]
$$

where $Y^{E L}$ is the general electric admittance, $L$ is a diagonal matrix of piezoelectric lengths, $A$ is the diagonal matrix of the surfaces areas, and $s$ is the Laplace parameter. The general electrical admittance relation starts from the following sum of admittances

$$
Y^{E L}=Y^{D}+Y^{S H}
$$


where $Y^{D}$ is the admittance of the PZT and $Y^{S H}$ is the admittance of the shunt circuit coupled to the PZT. Admittances are inversely proportional to impedances $Y^{E L}=1 / Z^{E L}$, being dependent on the voltage and current ratio involved in the system $Z=V / I$. Based on this information, piezoelectric compliance with the shunt circuit produces the following relationship between mechanical stresses and strains

$$
s_{j j}^{S H}=s_{i j}^{E}\left[1-k_{i j}^{2} \bar{Z}_{i}^{E L}\right]
$$

where $s^{S H}$ is the compliance of the shunt circuit coupled to the PZT, $\bar{Z}_{i}^{E L}$ is the matrix of non-dimensional electrical impedance obtained by dividing the general impedance $Z^{E L}$ by the impedance $Z^{D}$, and $k_{i j}=$ $d_{i j} \sqrt{s_{j j}^{E} \in \in_{i}^{T}}$ is the electromechanical coupling that represents the percentage of mechanical energy converted into electrical energy. The Young's modulus of a PZT with shunt circuit is dependent on the coupling relationships and the impedances involved. The relation to Young's modules loaded in direction $j$ and deflected through direction $i$ can be expressed as

$$
E_{j j}^{S H}=E_{j j}^{D} \frac{1-k_{i j}^{2}}{1-k_{i j}^{2} \bar{Z}_{i}^{E L}}
$$

where $E_{j j}^{S H}$ is the Young's modulus of the shunt circuit and $E_{j j}^{D}$ is the Young's modulus of the PZT.

\subsection{Shunt circuits and impedances variations}

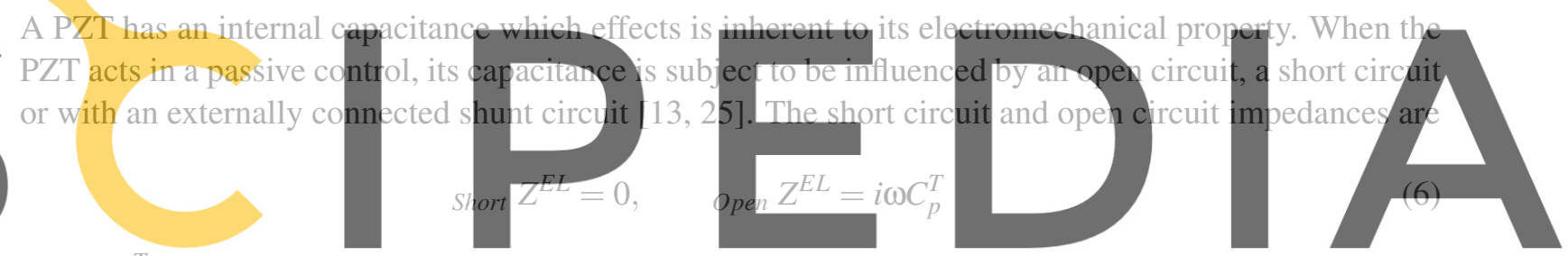

where $C_{p}^{T}$ is the capacitance between the PZT surfaces perpendicular to the direction $i$ (in constant stress).

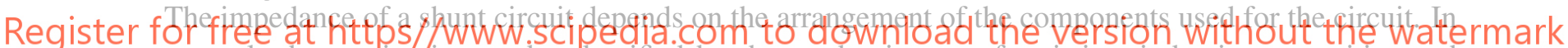
general, shunts circuits can be classified by the predominance of resistive, inductive, capacitive and

switching elements. The impedances of the shunt circuit are associated with a resonance frequency, for circuits with predominant resistive components the coupling factor is considered $\omega_{R}$, for circuits with predominant inductance we have the relation $\omega_{L}$.

$$
\omega_{R}=\sqrt{1-k_{i j}^{2}} / R C_{p}^{T}, \quad \omega_{L}=1 / \sqrt{L C_{p}^{T}}
$$

Figure 1 shows the main topographies of shunt circuits used to promote energy dissipation effects associated with the vibration, noise and wave propagation attenuation. 


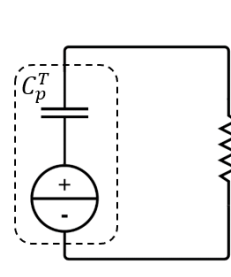

(a)

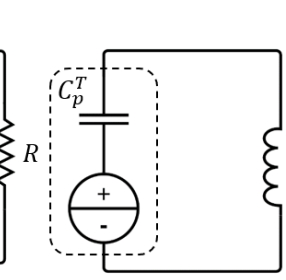

(b)

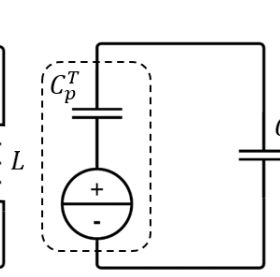

(c)

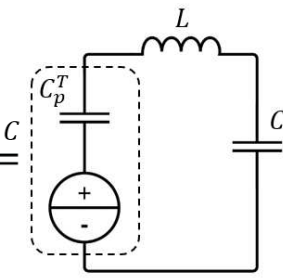

(d)

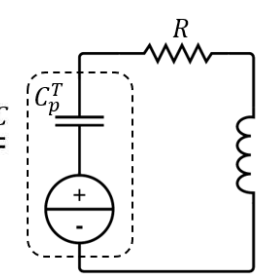

(e)

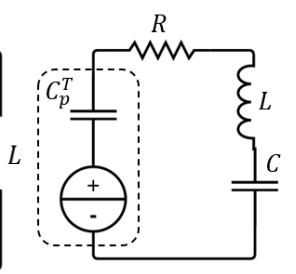

(f)

Figure 1: Shunt circuit topographies a) pure resistive; b) pure inductive; c) pure capacitive; d) LC; e) RL; f) RLC.

Figure 1, everything inside the dashed line is the piezoelectric representation and everything outside are the elements that make up the shunt circuit. Figure 1a shows a pure resistive shunt circuit, where, according to Eq.3, the piezoelectric admittance $Y^{D}=i \omega C_{p}^{T}$ is added to the resistor's influence for the following general impedance ratio

$$
Z^{E L}=\frac{1}{i \omega C_{p}^{T}+\frac{1}{R}}
$$

For the purely inductive circuit, we have the admittance ratio $Y^{S H}=1 / i \omega L$, where the real part is zero. Therefore, the general impedance ratio for the circuit in Figure $1 \mathrm{~b}$ is
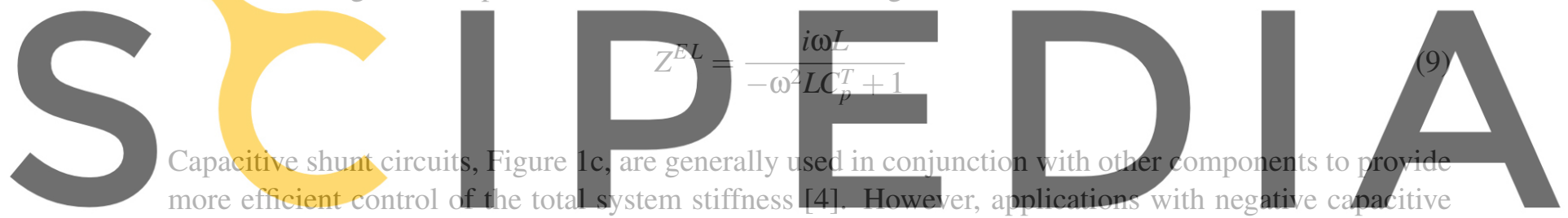

elements are being addressed, as they promote attenuation and bandgap effects in periodic structures [3].

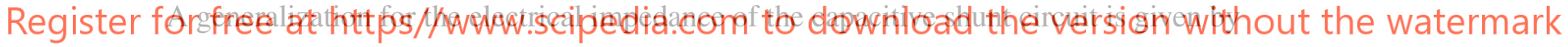

$$
Z^{E L}=\frac{1}{i \omega C_{p}^{T}}
$$

For circuits with inductive and capacitive elements, a predominant imaginary complex is established as a pass-through filter. In the circuit of Figure 1d, a control range associated with the frequency with $Y^{S H}=1 /(i \omega L+1 / i \omega C)$ is presented for admittance in series and $Y^{S H}=1 / i \omega L+i \omega C$ in parallel. Based on this logic, the general impedance for the series and parallel circuit can be expressed as

$$
\text { Series } Z^{E L}=\frac{i \omega L+\frac{1}{i \omega C}}{-\omega^{2} L C_{p}^{T}+\frac{i \omega C_{p}^{T}}{i \omega C}+1}, \quad \text { Parallel } Z^{E L}=\frac{i \omega L}{1-\omega^{2} L C-\omega^{2} L C_{p}^{T}}
$$

The RL circuit in Figure 1e is one of the most used shunts to promote passive vibration control. The effects resulting from its coupling to periodic structures are similar to those of a mechanical absorber without damping. Forward [10] investigated the inductive element as an absorber and observed an equivalent behavior when adding extra mass to the system. However, the inductance is inversely proportional 
to the frequency of the oscillations to be attenuated. The general impedances associated with the admittances of the series $Y^{S H}=1 /(R+i \omega L)$ and parallel $Y^{S H}=1 / i \omega L+1 / R$ circuit are represented as follows

$$
\text { Series } Z^{E L}=\frac{R+i \omega L}{1-\omega^{2} L C_{p}^{T}+i \omega R C_{p}^{T}}, \quad \text { Parallel } Z^{E L}=\frac{i \omega R L}{i \omega L+R-\omega^{2} R L C_{p}^{T}}
$$

Similar to RL and LC circuits, the RLC shunt circuit, Figure 1f, is a complete version of electrical components with specific properties for attenuation and vibration control. The admittances for the series $Y^{S H}=1 /(R+i \omega L+1 / C)$ and parallel $Y^{S H}=1 / R+1 / i \omega L+i \omega C$ circuit form the following impedances

$$
\text { Series } Z^{E L}=\frac{R+i \omega L+\frac{1}{i \omega C}}{1+i \omega C_{p}^{T}\left(R+i \omega L+\frac{1}{i \omega C}\right)}, \quad \text { Parallel } Z^{E L}=\frac{i \omega L}{i \omega L\left(i \omega C_{p}^{T}+i \omega C\right)+\frac{i \omega L}{R}+1}
$$

There are other variations of shunting circuits, such as switched shunt circuits (active shunt), and multisource circuits, both of which are built with various electrical components that conduct specific vibrational control settings for the system [25].

\section{SPECTRAL ELEMENT OF SMART MATERIAL}

\subsection{Piezoelectric beam coupling}

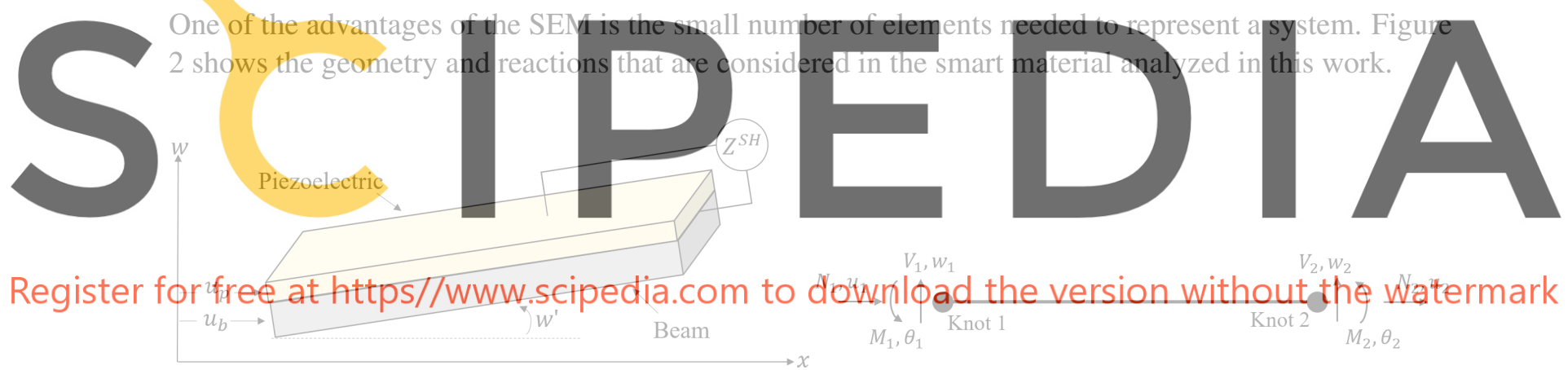

(a)

(b)

Figure 2: Beam smart representation: a) Physical structure; b) Spectral element.

Figure $2 \mathrm{~b}$ shows the smart beam material, and the two-node spectral element with each node has the nodal forces and displacements. An Euler-Bernoulli beam coupled with a bar which model de PZT transducer layer is the smart beam material $[9,18]$. The thickness and displacement relationships of the beam with the PZT are defined by

$$
u_{p}=u_{b}-\frac{h}{2} \frac{\partial w}{\partial x}
$$

where $u_{p}$ and $u_{b}$ are the axial displacements in the piezoelectric and the beam, respectively. The transverse displacement is represented by $w$, and $h=h_{p}+h_{b}$ is the sum of the thickness of the PZT with 
that of the beam. By following the Hamilton's the beam and bar coupled equations of motion with with Young's modulus $E$, mass density $\rho$ and damping $c$, are given as follows

$$
\begin{array}{r}
E I w^{\prime \prime \prime \prime}+\rho A \ddot{w}+c A \ddot{w}=-\alpha \ddot{u}_{b}^{\prime}+\beta u^{\prime \prime \prime}+\lambda \ddot{w}^{\prime \prime}+c_{1} \ddot{w}^{\prime \prime}-c_{4} \ddot{u}_{b}^{\prime}+F w^{\prime \prime}+p(x, t) \\
E A u_{b}^{\prime \prime}-\rho A \ddot{u}_{b}-c A \ddot{u}_{b}=-\alpha \ddot{w}^{\prime}+\beta w^{\prime \prime \prime}-\tau(x, t)
\end{array}
$$

where

$$
\begin{gathered}
E A=E_{b} A_{b}+E_{p} A_{p}, \quad E I=E_{b} I_{b}+E_{p} I_{p}+(1 / 4) E_{p} A_{p} h^{2}, \quad c_{4}=(1 / 2) c_{p} A_{p} h, \\
\rho A=\rho_{b} A_{b}+\rho_{p} A_{p}, \quad E_{p}=C_{11}^{D}-h_{31}^{2} \beta_{33}^{S-1}, \quad c A=c_{b} A_{b}+c_{p} A_{p}, \\
\gamma=(1 / 4) \rho_{p} A_{p} h^{2}, \quad \alpha=(1 / 2) \rho_{p} A_{p} h, \quad \beta=(1 / 2) E_{p} A_{p} h, \quad c_{1}=(1 / 4) c_{p} A_{p} h^{2}
\end{gathered}
$$

Since SEM is formulated in the frequency domain the spectral element assumption is that displacements and voltage $V(t)$, are expressed in a spectral form by

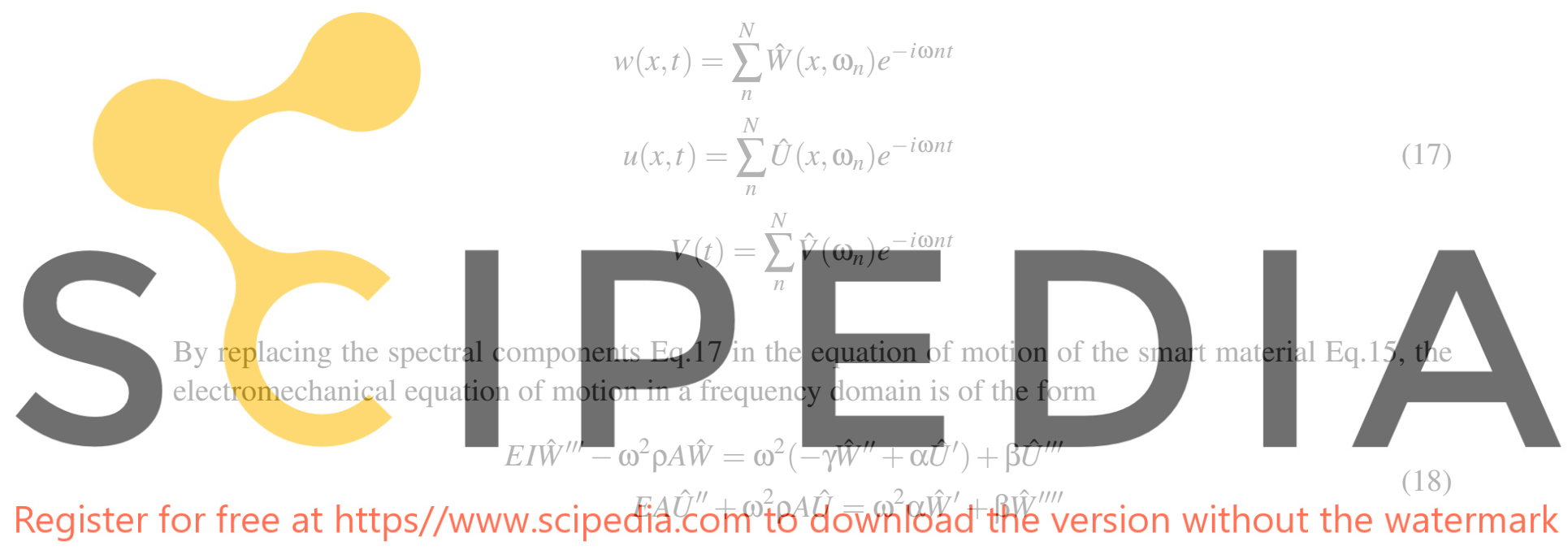

The solution of the equations of motion as a function of frequency can be performed from a general solution for the components of spectral displacements with the following relationship

$$
\begin{array}{r}
\hat{W}(x)=\sum_{i=1}^{6} a_{j} e^{-i k_{j} x}=g(x, \omega) a \\
\hat{U}(x)=\sum_{i=1}^{6} r_{j} a_{j} e^{-i k_{j} x}=g(x, \omega) R a
\end{array}
$$

where

$$
\begin{aligned}
& g(x, \omega)=\left[e^{-i k_{1} x} e^{-i k_{2} x} e^{-i k_{3} x} e^{-i k_{4} x} e^{-i k_{5} x} e^{-i k_{6} x}\right] \\
& a=\left[\begin{array}{llllll}
a_{1} & a_{2} & a_{3} & a_{4} & a_{5} & a_{6}
\end{array}\right]^{T} \\
& R=\operatorname{diag}\left(\frac{-\omega k_{j} c_{4}-i \omega^{2} k_{j} \alpha+i k_{j}^{3} \beta}{-k_{j}^{2} E A+\omega^{2} \rho A-i \omega c A}\right)
\end{aligned}
$$


The eigenvalue problem is obtained from characteristic equation of the smart material motion equation gives the wavenumbers $k_{i}(i=1,2,3 . ., 6)$ determined as

$$
\begin{gathered}
\left(\beta^{2}-E A E I\right) k^{6}+\omega^{2} L^{2}(2 \alpha \beta-\rho A E I A E I-\lambda E A) k^{4}+\omega^{2} L^{4}\left(\alpha^{2} \omega^{4}+\right. \\
\left.\rho A\left(E A-\lambda \omega^{2}\right)\right) k^{2}+\omega^{4} L^{6} \rho A^{2}=0
\end{gathered}
$$

By considering a finite structure of length $L$, the spectral nodal displacements in terms of $k_{j}$, satisfying the boundary conditions of the system, can be defined by

$$
x=\mathbf{H}(\omega) a=\left[\hat{U}_{1} \hat{W}_{1} \hat{\theta}_{1} \hat{U}_{2} \hat{W}_{2} \hat{\theta}_{2}\right]^{T}
$$

where
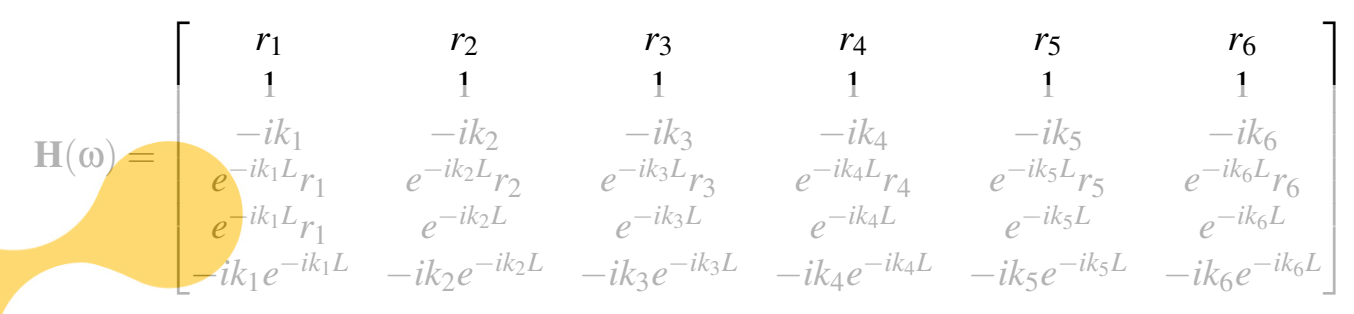

From the dynamic interaction of each nodal component, the following dynamic stiffness matrix of the spectral beam smart element is
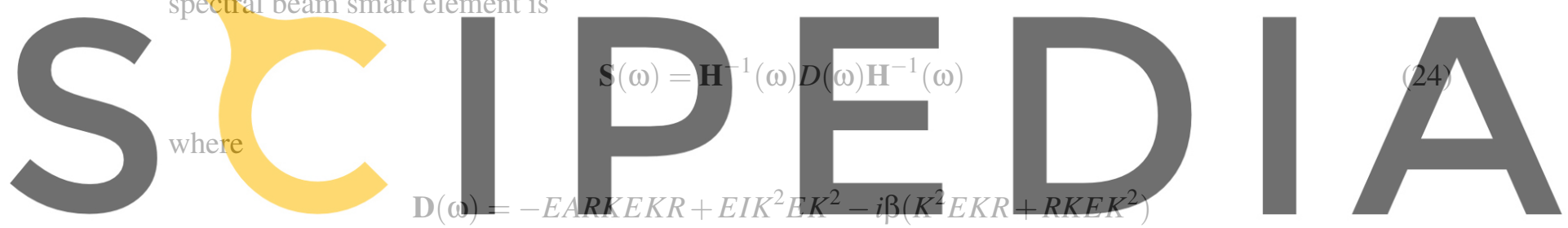

Register for free at https//www scipe $\omega^{2}[\rho A(E+R E R)+i \alpha(K E R+R E K)-\lambda K E K]$

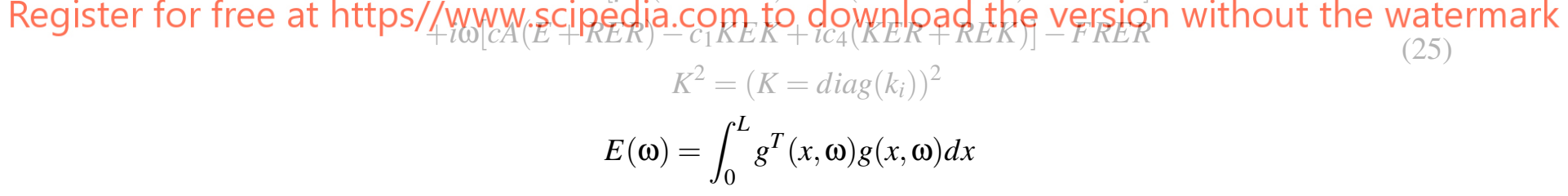

The dynamic relationship between the beam and the PZT layer is given by $\mathbf{S}(\omega)$.

\subsection{Piezoelectric beam coupling with shunt circuit}

The global electromechanical equation of motion that couple the shunt circuit to the PZT is defined in terms of the spectral stiffness matrix with,

$$
\begin{array}{r}
\mathbf{S}(\omega) x-\mathbf{S}_{S H}(\omega) V(\omega)=f(\omega) \\
i \omega \mathbf{S}_{S H}(\omega) x+i \omega C_{p}^{T} V(\omega)=I(\omega)
\end{array}
$$

where $\mathbf{S}_{S H}$ is the spectral stiffness matrix of the shunt circuit, $x$ is the generalized nodal displacement, $f$ the generalized force, $I$ is the current in the spectral domain, and $V$ the voltage. Similar to the nodal 
relation in Eq. 22, the behavior of the piezoelectric with the branch circuit can be expressed with the displacements and equivalent nodal forces. Therefore, the spectral stiffness matrix for the shunt circuit can be assembled as follows

$$
\mathbf{S}_{S H}(\omega)=\left[N_{e 1} \hat{W}\left(x_{0}, \omega\right), 0,-M_{e 1} \hat{W}\left(x_{0}, \omega\right),-N_{e 2} \hat{W}\left(x_{0}, \omega\right), 0, M_{e 2} \hat{W}\left(x_{0}, \omega\right)\right]^{T}
$$

where

$$
N_{e 1}=N_{e 2}=\frac{k_{i j}^{2} i \omega Z^{E L} b_{p} d_{31} E_{p}}{1+i \omega C_{p}^{T} Z^{E L}} \quad M_{e 1}=M_{e 2}=\frac{k_{i j}^{2} i \omega Z^{E L} h b_{p} d_{31} E_{p}}{2+2 i \omega C_{p}^{T} Z^{E L}}
$$

The PZT's nodal functions with shunt circuit are related to PZT coupling coefficient $k_{i j}$, the width $b_{p}$, Young's modulus $E_{p}$, and to piezoelectric constants $d_{31}$. Thus, a general representation for the dynamic behaviour of an intelligent material is shown with the following expression

$$
\left[\mathrm{S}(\omega)+\omega^{2} \mathrm{~S}_{S H}^{2}\left(\frac{1}{i \omega+1 / Z^{E L}}\right)\right] x(\omega)=f(\omega)
$$

where $Z^{E L}$ is the associated impedance for each shunt circuit configuration.

\section{NUMERICAL ANALYSIS}

The numerical simulation demonstrates the dynamic analysis of an aluminium smart beam coupled to a piezoelectric with differen electric material has a d coefficient $k_{31}=0.31$,
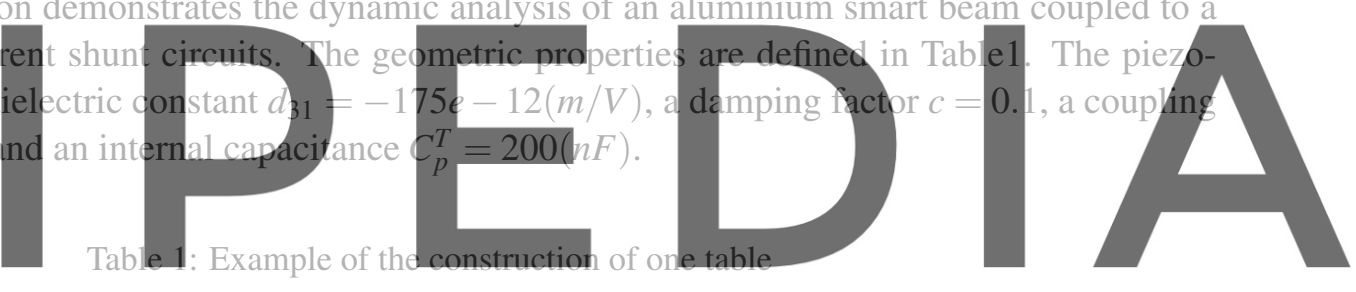

Properties Beam Properties Piezoelectric
Register for free at https//Wwy

\begin{tabular}{ccc}
\hline Width $[\mathrm{mm}]$ & 12.7 & 12.7 \\
\hline Thickness $[\mathrm{mm}]$ & 2.286 & 0.762 \\
\hline Young's modulus $[\mathrm{GPa}]$ & 71 & 64.9 \\
\hline Density $\left[\mathrm{kg} / \mathrm{m}^{3}\right]$ & 2700 & 7600 \\
\hline
\end{tabular}

Figure 3 shows the general impedances of each shunt configuration provided to the smart material. 


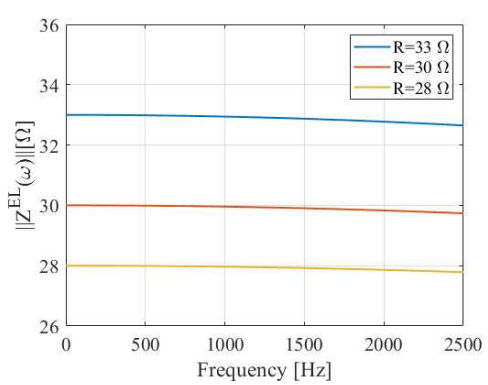

(a)
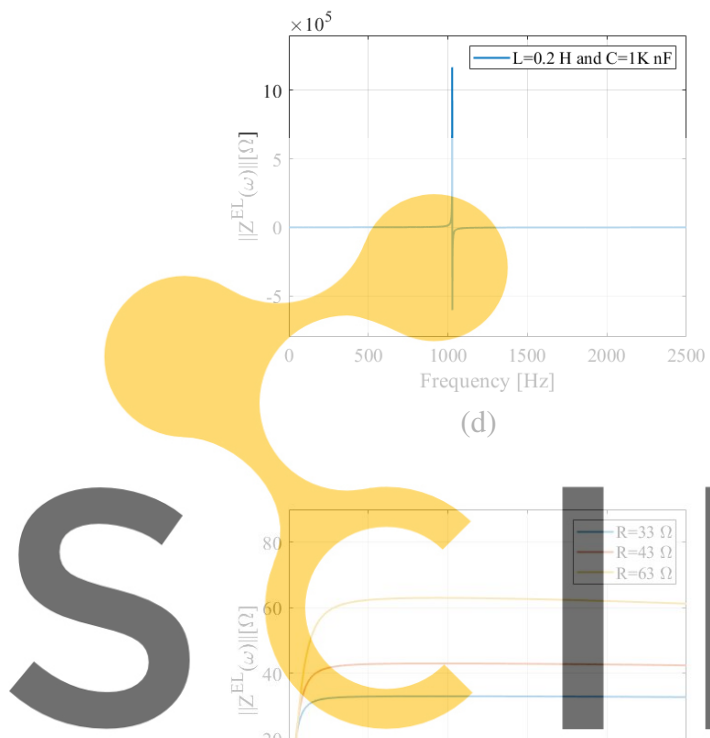

Register for free at https/:/www.scipedia.com

(g)

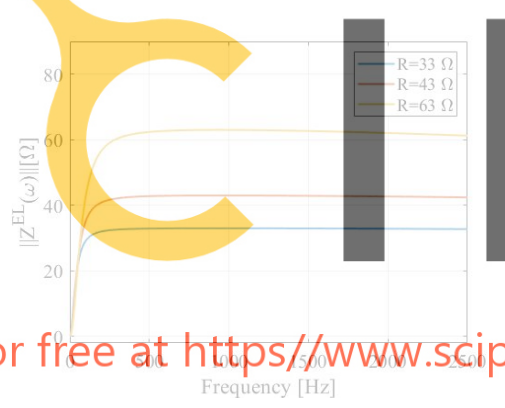

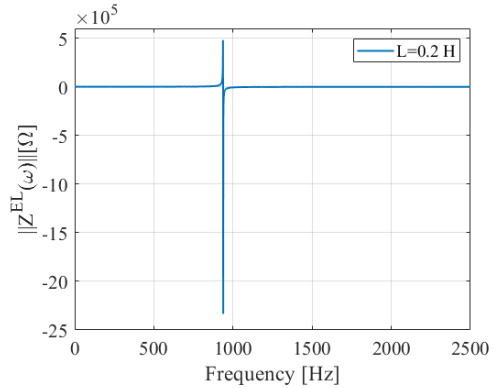

(b)

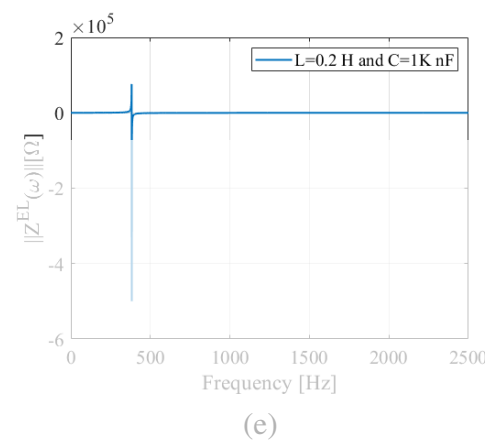

(e)
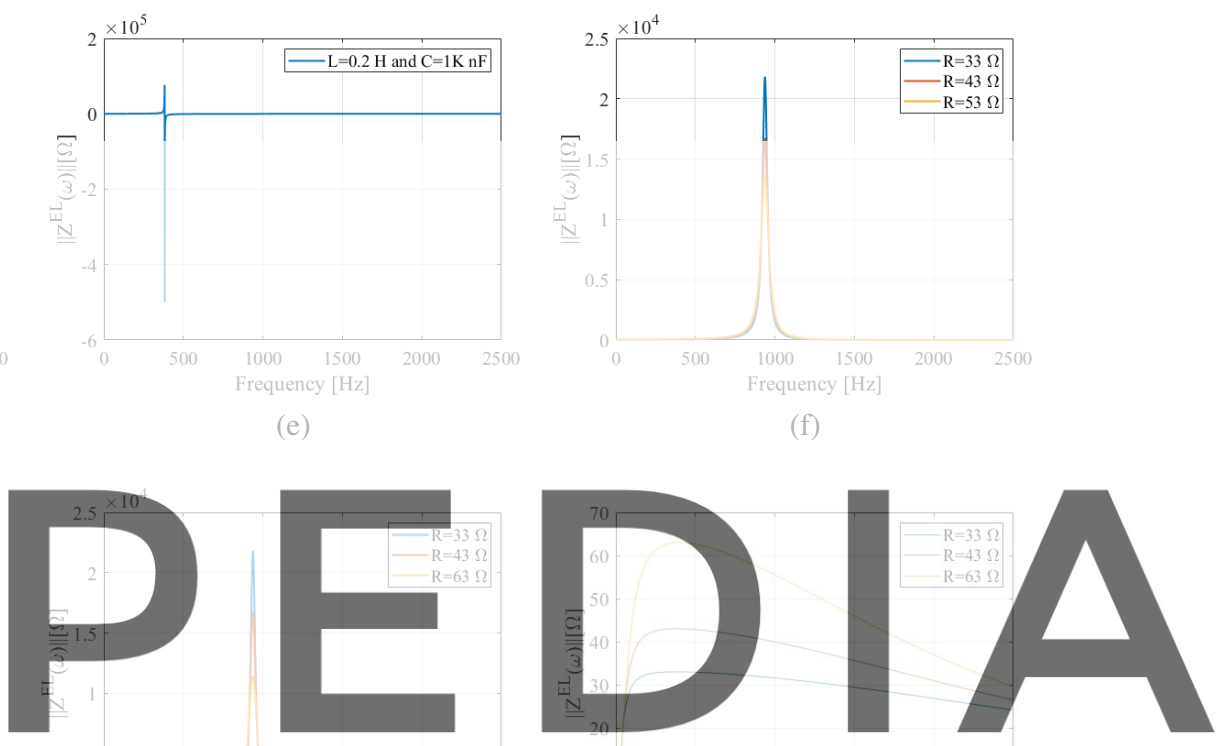

(f)

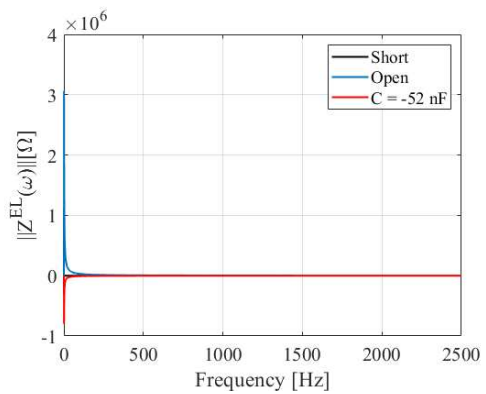

(c)

\section{(h}

download sthe version without the watermark

(i)

Figure 3: Impedances of Smart Material for shunt circuit: a) pure R (real part); b) pure L (imaginary part); c) short, open and negative capacitance (imaginary part); d) LC series (imaginary part); e) LC parallel (imaginary part); f) RL series (real part); g) RL parallel (real part); h) RLC series (real part); i) RLC parallel (real part).

The resistive shunt circuit's impedance when coupled to the PZT induce a damping effect on the structure. Figure 3a shows that the higher the resistance greater is the dissipation. The inductive circuit showed in Figure 3b, the dissipation effect appears at target resonant frequency. The resistive shunt circuit's impedance when coupled to the PZT induce a damping effect on the structure. Figure 3a shows that for higher the resistance greater is the dissipation. The inductive circuit showed in Figure 3b, the dissipation effect appears at target resonant frequency. The impedance in Figure $3 \mathrm{c}$ represents a negative capacitance of a shunt circuit, where the relationship with the resistance is imposed with $Z^{S H}=-R / i \omega C_{n e g}$. The capacitive shunts circuits influence the structural rigidity and are dependent on the resonant frequency. This dependency is also presented in the LC circuit. However, its impedances tend to more inductive 
behaviour for the topography in series (Figure 3d), and more capacitive for the topography in parallel (Figure 3e). The shunt circuits RL and RLC of Figures 3f, 3g, 3h and 3i, show similar impedance when associated with alike topographies. The RL circuit, also known as resonant circuits, converts to an RLC circuit coupled with a PZT. The difference between them is adjusting stiffness, with RL limited to $C_{p}^{T}$ and RLC limited to its own capacitor $C$, which can compromise the appropriate value to adjust the desired stiffness. Figure 4 shows the frequency response functions in the smart material's free-free boundary condition associated with shunt circuits' different couplings.

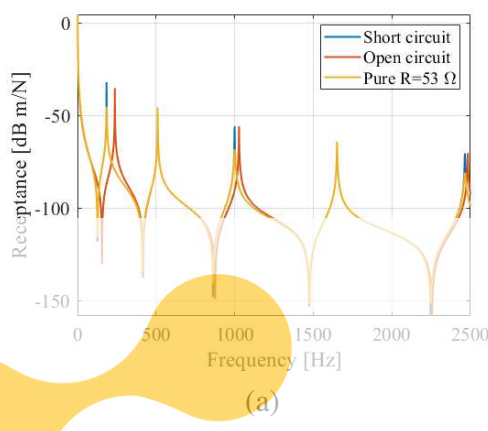

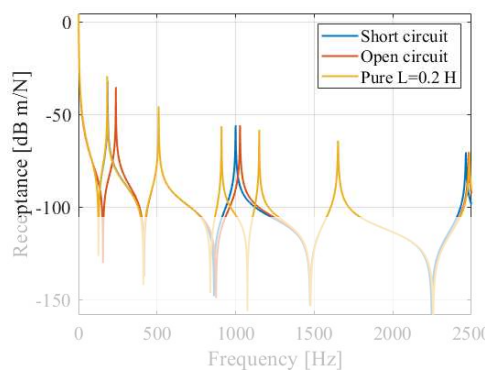

(b)

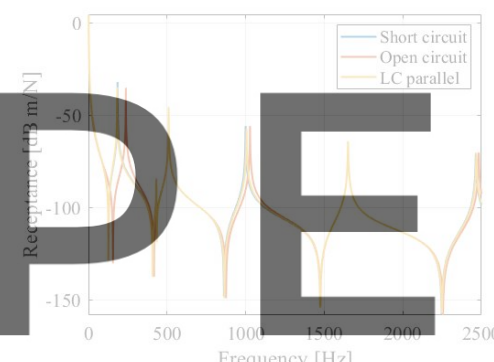

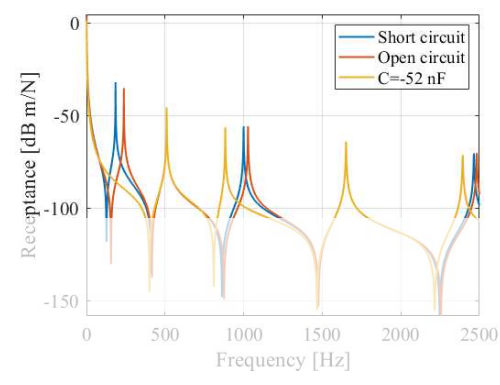

(c)

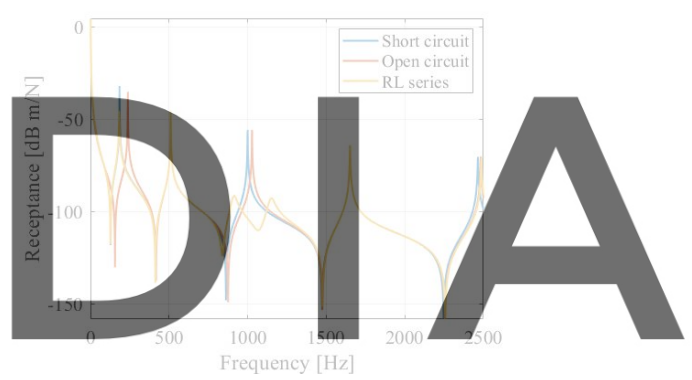

Register for free at https///www.scipedia.com to (e) download the version without the watermark

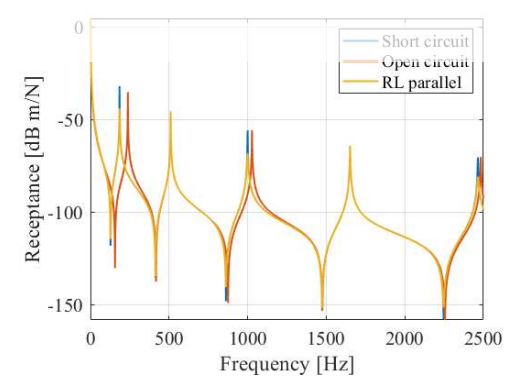

(g)

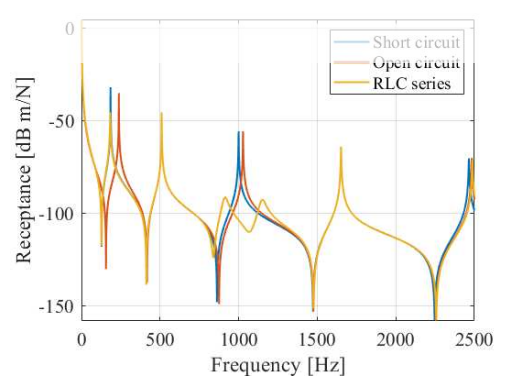

(h)

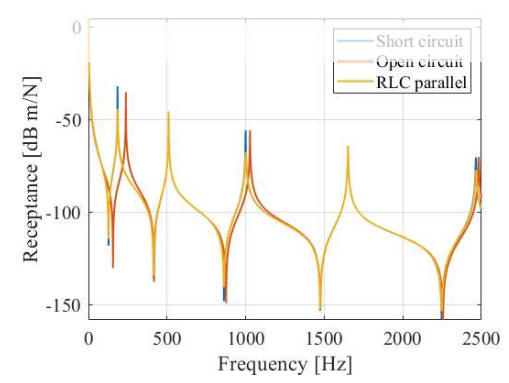

(i)

Figure 4: Frequency Response Function of smart material for shunt circuit configuration: a) Pure R; b) Pure L; c) Pure C; d) LC series; e) LC parallel; f) RL series; g) RL parallel; h) RLC series; i) RLC parallel.

Figure $4 \mathrm{a}$, the coupling of a resistive shunt circuit is able to dampen the resonance peaks of the intelligent material. The damping location $(1000 \mathrm{~Hz})$ is defined according to the coupling coefficient $k_{i j}$ and the 
piezoelectric capacitance $C_{p}^{T}$, while the resistance value determines the damping size. Figure $4 \mathrm{~b}$,shows the receptance response of the smart beam changes similar to the addition of mass on the structure. The resonance peak located at the frequency of $1000 \mathrm{~Hz}$ is divided into two with close amplitudes, also seen in the cases of the LC circuit series topography (Figure 4d), RL (Figure 4f) and RLC (Figure 4h). Hence, the resistor circuit component influence direct in the resonance peaks and the smart beam vibration damping. The responses influenced by the circuit shunts connected to a capacitive element change the stiffness of the structure. Figure 4c, the firsts resonance peaks of the smart beam, between 0 and $500 \mathrm{~Hz}$, are eliminated when a negative capacitance of the circuit operated on short and open circuits condition. In the other circuits (Figure $4 \mathrm{~d}, 4 \mathrm{e}, 4 \mathrm{f}, 4 \mathrm{~g}, 4 \mathrm{~h}$ and $4 \mathrm{i}$ ) the positive capacitance of $C=1 \mathrm{~K} \mathrm{nF}$ is used, leading to attenuation happening in a milder way.

\section{CONCLUSIONS}

In this article, vibration attenuation was performed by the use of circuit shunts connected to a smart beam consisting of an aluminium beam coupled to a PZT layer. The PZT transducer is connected to different topographies of external shunts circuits, and the effect of each one in the vibration attenuation demonstrated. SEM was used to model the system and estimate the smart beam receptance response. The shunt circuits used resistor, inductor and capacitor in their electrical configuration. Once the shunt was connected to the structure, the receptance FRF shows each importance's influence in the response. The smart beam connected to an inductive shunt induces similar attenuation when added mass in the system. The resistive shunt input structural damping over the beam and the capacitive shunt added some rigidity in the smart material. In all cases, the shunt circuit has a great influence on the smart beam response.

\section{REFERENCES}

[1] Airoldi, L., and Ruzzene, M. Design of tunable acoustic metamaterials through periodic arrays of resonant shunted piezos. New Journal of Physics 13 (2011), Art. no. 113010.

[2] Barbosa, B., Machado, M. Analysis of vibration attenuation in a beam coupled with a piezoelectric in shunt configuration using the spectral element method. COBEM, Uberlândia-MG, 2019.

[3] Chen, Y. Y., Huang, G. L., and Sun, C. T. "Band Gap Control in an Active Elastic Metamaterial With Negative Capacitance Piezoelectric Shunting.” ASME. J. Vib. Acoust. 2014; 136(6): 061008.

[4] Cheng, C. H. 2007. Analysis and Experiment for the Deflection of a Shear-mode PZT Actuator. Smart Materials and Structures, v. 16, n. 1, p. 230.

[5] Davis, C.L., Lesieutre, G.A., 2000, "An actively tuned solid-state vibration absorber using capacitive shunting of piezoelectric stiffness", Journal of Sound and Vibration, Vol 232, pp.601-617.

[6] Dutkiewicz, M., and Machado, M.R., 2019, Spectral Approach in Vibrations of Overhead Transmission Lines, IOP Conf. Ser.: Mater. Sci. Eng., 471,p. 052029.

[7] Dutkiewicz, M., and Machado, M. R., 2019, Dynamic Response of Overhead Transmission Line in Turbulent Wind Flow With Application of the Spectral Element Method, IOP Conf. Ser.: Mater. Sci. Eng., 471, p. 052031.

[8] Dutkiewicz, M. and Machado, M.R. 2019. Spectral element method in the analysis of vibrations of overhead transmission line in damping environment. Struct. Eng. and Mechanics, 71(3),291-303. 
[9] Doyle, J. F. Wave Propagation in Structures: a spectral analysis approach. 2. ed. New York: Springer-Verlag, 1997.

[10] Forward, R. L., 1979, "Electronic Damping of Vibrations in Optical Structures," J. Appl. Opt., 18(5), pp. 690-697.

[11] Gripp, J. A., and Rade, D. A. Vibration and Noise Control Using Shunted Piezoelectric Transducers: A review". Mechanical Systems and Signal Processing 112. (2018). 359-383.

[12] Gonella, S. and Ruzzene, M. 2008. Homogenisation of vibrating periodic lattice structures, Appl. Math. Model. 32, 459-482. doi:10.1016/j.apm.2006.12.014.

[13] Hagood, N. W., and Flotow, A. V., 1991, "Damping of Structural Vibrations With Piezoelectric Materialsand Passive Electrical Networks," J. Sound Vib., 146(2), pp. 243-268.

[14] Hussein, M. L., Leamy, M. J. and Ruzzene, M. 2014. Dynamics of Phononic Materials and Structures: Historical Origins, Recent Progress, and Future Outlook. Applied Mechanics, 66(4)-40802.

[15] B. JAFFE, R. COOK and H. JAFFE 1971 Piezoelecfric Ceramics. New York: Academic Press.

[16] James B. Min, *Kirsten P. Duffy, Andrew J. Provenza. Shunted Piezoelectric Vibration Damping Analysis Including Centrifugal Loading Effects. 2011.https://ntrs.nasa.gov/citations/20110016111

[17] K Yi, M Collet, M Ichchou and L Li. Flexural waves focusing through shunted piezoelectric patches. Smart Materials and Structures. 2016. doi:10.1088/0964-1726/25/7/075007

[18] Lee, U. 2009, Spectral Element Method in Structural Dynamics, John Wiley Sons, Asia.

[19] Lee, U. and Kim, J., 2000. "Dynamics of elastic-piezoelectric two-layer beams using spectral element method.”. International Journal of Solids and Structures, 37, pp. 4403-4417.

[20] Leo, D.J. 2007. Engineering Analysis of Smart Material Systems, JohnWiley Sons, NJ, p.1-7.

[21] Machado, M. R., Fabro, A. T., and Moura, B. B., 2019. "Flexural waves propagation in piezoelectric metamaterial beam". In Proceedings of 15th INTERNATIONAL CONFERENCE Dynamical Systems - Theory and Applications, Lodz, Poland.

[22] Moura, B. B., Borges, M. C., and Machado, M. R. (2020). Vibration and Wave Propagation Control in a Smart Metamaterial Beam with Periodic Arrays of Shunted Piezoelectric Patches. 49th International Congress and Exposition on Noise Control Engineering INTER-NOISE. Seoul, Korea.

[23] Machado, M. R., Fabro, A. T., and Moura, B. B., 2019. "Spectral element approach for flexural waves control in smart material beam with single and multiple resonant impedance shunt circuit". Journal of Computational and Nonlinear Dynamics, doi: https://doi.org/10.1115/1.4047389.

[24] Park, C. H. Vibration Control of Beams with Negative Capacitive Shunting of Interdigital Electrode Piezoceramics J. Vib. Control (2005) 11:331-46.

[25] Santana, D. C., Rade, D. A., and Steffen, V. Estudo de Técnicas de Controle de Vibração Empregando Piezocerâmicas Combinadas com Circuitos Shunt. 13 POSMEC - Simpósio do Programa de Pós-Graduação em Engenharia Mecânica. FEMEC/UFU, Uberlândia. (2003).

[26] Yan, B., Wang, K., Hu, Z., Wu, C. and Zhang, X. Shunt Damping Vibration Control Technology: A Review. Applied Sciences, 7(5), May, p. 494. (2017). 